Michalina Błażkiewicz

Ida Wiszomirska

Katarzyna Kaczmarczyk

Andrzej Wit

\title{
TYPES OF FALLS AND STRATEGIES FOR MAINTAINING STABILITY ON AN UNSTABLE SURFACE
}

\author{
Józef Piłsudski University of Physical Education, Warszawa, Poland
}

Faculty of Rehabilitation

\begin{abstract}
Background: Falls constitute an important health issue. They cause significant morbidity, mortality and have marked psychological effects on the individual, too. The aim of this study has been to determine parameters describing human movement strategies for balance and the reaction if balance is lost as a result of an unstable ground, and to attempt to describe the types of falls. Material and Methods: The study group comprised 20 volunteers. Kinematic parameters of falling and dynamic stability were measured using the Vicon Motion System and the Biodex Balance System SD. During the test, subjects stood for $20 \mathrm{~s}$ on the tilting platform. The analysis was conducted based on the first recordings, when the participants were not prepared for the event and their reactions were natural. A cluster analysis tool was applied to divide the behavior of people during the test. Results: Based on motion range for kinematic parameters, the cluster analysis revealed 2 types of human behavior: falling (stepping) and restoring balance. Two types of falls were also observed: side and back falls. Moreover, on the basis of angular values for tilting plate, 4 zones were determined. The frequency of access to these zones by each joint revealed 3 strategies to maintain balance: ankle, knee and hip strategy. Conclusions: A set of initial conditions that may be used for future numerical simulations was also determined. Furthermore, the results presented in this study are likely to support the analysis of the effects and the falling patterns. Med Pr 2018;69(3):245-252

Key words: falls, postural stability, biomechanics, cluster analysis, range of motion, loss of balance

Corresponding author: Michalina Błażkiewicz, Józef Piłsudski University of Physical Education, Faculty of Rehabilitation, Marymoncka 34, 00-968 Warszawa, Poland, e-mail: michalinablazkiewicz@gmail.com

Received: April 24, 2017, accepted: November 30, 2017
\end{abstract}

\section{INTRODUCTION}

Postural control may be defined as the postural strategies used for maintaining the vertical projection of the center of mass of the body (CoM) within the base of support, that require recovery response strategies when exposed to perturbations in order to prevent falls [1]. Falls have been reported as one of the leading causes of injuries [2] and are defined as uncontrollable body displacements which cannot be adjusted within a specific time. With medical definition, falls are considered to be unintended changes in body position, that consist in losing balance during walking or other activities that lead to the situation where the person lands on the ground or another low-level surface.

The most frequent events that lead to fall episodes include: losing balance due to insufficient stability of the physical factor, slipping, trips, climbing on poles, scaffoldings and other structures. With a substantial number of falls in both young and older people [3], numerical methods have been applied to recreate events connected with the threat of mechanical injuries $[4,5]$. An important factor that has an effect on the falling trajectory is human reaction at the instant of losing balance. The falling human determines initial conditions that represent the input data for starting the simulation. Major areas of focus will include how, why, and when a person is likely to fall, the direction of fall, body's final resting position, the part of the body most likely to be injured based on the ground and body impact sites [6].

Balance recovery strategies are divided in the literature [7] into balance recovery through: sway (ankle or hip strategy), a single step or more steps. The ankle strategy is characteristic of repositioning the CoM by moving the whole body as a single-segment inverted pendulum by production of torque at the ankle. In contrast, the hip

Funding: this work was supported by the Polish National Science Centre (grant No. 2011/01/D/N27/05296 entitled "Mathematical modeling of compensatory functions of lower limb muscles during normal and pathological gait," project manager: Michalina Błażkiewicz, Assoc. Prof.). 
strategy moves the body as a double-segment inverted pendulum with counter phase motion at the ankle and hip. When violent disruption of balance over-powers the ankle and hip strategies, 2 falling strategies are reported [7]: partial fall involving contact to one or both knees and/or hands and complete fall involving contact to the trunk and/or pelvis. However, there are no descriptions of human body reaction during the fall caused by instability of the physical factor under the lower limbs. Slipping and no possibility to recover the stability causes the body backward or lateral motion, which might lead to the hitting with the pelvis against the ground, followed by the head. Therefore, the aim of this study has been to determine parameters that describe human reaction during the moment of losing balance and to attempt to separate parameters responsible for losing the stability and allowing for categorization of the types of reactions adopted by people standing on the unstable ground.

\section{MATERIAL AND METHODS}

\section{Participants and measurements}

Twenty male volunteers (body mass: $68.57 \pm 10.68 \mathrm{~kg}$, body height: $174.5 \pm 8.48 \mathrm{~cm}$, age: $24.07 \pm 7.32$ years old) participated in this study. No participants reported any sensory impairment or physical injury that hindered performance of the balance task, nor had any of the participants previous experience with balance training. In addition, none of the participants was engaged in recreational and professional sports. Before the measurement, the participants were informed about the procedures used and the possibility of stopping the experiment at any moment. All participants signed informed consent forms before participation, in accordance with the local ethics committee at Józef Piłsudski University of Physical Education in Warszawa, Poland.

The study consisted of removal from the equilibrium position of a subject located $1880 \mathrm{~mm}$ above the ground. For this purpose, the tilting platform Biodex Balance System (BBS) Static and Dynamic (SD) (Biodex, USA) was used. It comprised a circular unstable platform of $550 \mathrm{~mm}$ in diameter. The base of the plate was $1120 \mathrm{~mm}$ in width and $760 \mathrm{~mm}$ in length. Moreover, the BBS plate could concurrently be moved in the anterior-posterior and medial-lateral directions. Eight springs located underneath the outer edge of the platform provided the resistance to movement i.e., stability level of the platform. Setting 1 represented the least stable platform and setting 12 - the greatest platform stability.
In the initial state, the platform was positioned parallel to the ground and locked in the second position.

The participants stood on the BBS on their 2 legs. All trials were performed barefoot while foot position was recorded using the coordinates on the platform's grid. The standard software configuration (one $20 \mathrm{~s}$ trial) was used. A test was recorded once for each participant, whether the participant maintained balance or fell after the test time elapsed. A single test was used in order to reduce the potential effects of learning and fatigue. At the same time, a motion capture system (Vicon Motion Systems Ltd, United Kingdom), consisting of 9 infra-red cameras, was employed to collect kinematics data at a sampling rate of $100 \mathrm{~Hz}$. First, anthropometric measurements were taken for each person. Next, 34 spherical reflective markers were placed at anatomical landmarks according to the biomechanical model available within the motion capture system. Both systems had been calibrated according to the manufacturers' recommendations before the trials were conducted.

\section{Data analysis - parameters and variables}

The analysis was conducted based on the first recordings, when the participants were not prepared for the event and their reactions were natural. The range of motion ( $\mathrm{Ri}$ ) was computed for all kinematics variables in all planes using the following formula:

where:

$$
\mathrm{Ri}=\alpha_{\mathrm{i}, \max }-\alpha_{i, \min }
$$

$\alpha_{i, \max }-$ maximum values obtained during the test trial for i-joint and for CoM,

$\alpha_{i, \text { min. }}-$ minimum values obtained during the test trial for i-joint and for CoM.

From the degrees of tilt, the BSS values were calculated: the medial-lateral stability index (MLSI), the anterior-posterior stability index (APSI) and the overall stability index (OSI) [8]:

$$
\begin{gathered}
\text { APSI }=\sqrt{\frac{\sum(0-Y)^{2}}{\mathrm{~N}}} \\
\text { MLSI }=\sqrt{\frac{\sum(0-\mathrm{X})^{2}}{\mathrm{~N}}} \\
\text { OSI }=\sqrt{\frac{\sum(0-\mathrm{X})^{2}+\sum(0-\mathrm{Y})^{2}}{\mathrm{~N}}}
\end{gathered}
$$


where:

$\mathrm{Y}$ - displacement in degrees, from level, for motion in the sagittal plane,

$\mathrm{X}$ - displacement in degrees, from level, for motion in the frontal plane,

$\mathrm{N}$ - number of samples.

These indexes are standard deviations used for assessing fluctuations around the zero point. The MLSI and the APSI assess the deviations from the horizontal position on the AP and ML axes of the BSS, respectively. In contrast, the OSI is a composite of the MLSI and APSI and, thus, is sensitive to changes in both directions [9].

\section{Cluster analysis and categories of falls}

A cluster analysis tool was applied to divide the behavior of people during the test. The $14 \times 21$ matrix was used in the analysis. Twenty-one variables described movements of 14 participants in each plane. The parameters were chosen based on the highest values of the parameters of the range of motion (Ri). Therefore, the description was performed based on the CoM, hip, knee and ankle angle in the tree plane for all 14 participants.

The cluster analysis is a method of unsupervised classification and grouped elements in relatively uniform classes. The basis for grouping in the most of algorithms is similarity between elements. The k-means algorithm was used. The k-means methodology belongs to optimization-iteration methodologies and consists in dividing the whole set of objects according to the general principle of maximization of variances between individual groups while maximizing variances inside the groups studied. The k-means methodology may be described with several points. The starting point is the initial arbitrary division of the set into k-clusters.

The assignment of objects to groups should be performed so that maximal probability is attained with maximal intergroup differences. The algorithm is used until the items are divided so that the most significant results of the analysis of variances are obtained. The mean (i.e., centroid position) is calculated for each group. Next, using the conventional Euclidean metric, the distances between the first unselected item and the centroid position of individual groups are determined and classified to the closest group. Next, new means are calculated. The algorithm is repeated until the convergence criterion is reached, which most often happens for the step at which affiliation of points to classes does not change.

\section{Strategies to maintain stability}

The statistical analysis was conducted using the Statistica software (StatSoft, Poland), with the a level set at 0.05 . Normality of measured and calculated data distribution was assessed using the Shapiro-Wilk test. In order to see which kinematic variable is responsible to the highest degree for maintaining stability in the group of people who did not fall, Pearson's correlation coefficients were calculated between the stability index OSI and Ri parameters.

In order to determine what the strategy to maintain stability on tilting plate was, the division into 4 zones: I, II, III, IV was performed based on Arnold and Schmitz, and Glave et al. $[9,10]$. The division was made for the group of people who did not fall during the test. The following angular values determined the individual zones. The first zone was between $-5^{\circ}$ and $5^{\circ}$, which was marked I: $(-5)-5^{\circ}$. The second zone was defined as II: $(-10)-(-5)^{\circ}$ and $5-10^{\circ}$, III: $(-15)-(-10)^{\circ}$ and $10-15^{\circ}$; IV: $(-\infty)-(-15)^{\circ}$ and $15^{\circ}-\infty$. With 20 s of the test, recorded at the frequency of $100 \mathrm{~Hz}$ for each joint, the number of times the joint remained within the zones I, II, III or IV was counted. The frequency of access to these zones by each joint may reveal proprioceptive incapacity associated with ankle or lower extremity pathology. Therefore, the one-way ANOVA was performed to test differences in time spent in each zone by each joint of lower limb.

\section{RESULTS}

\section{Cluster analysis}

On the basis of $\mathrm{Ri}$, calculated for the $14 \times 21$ matrix, the solution in the form of 3 clusters that grouped participants to the class of people with stable position (cluster 1), falling backwards (cluster 2) and falling to the side (cluster 3) was found after the first iteration (Figure 1).

Maximal mean for the cluster 1 (stability) is $173.06 \mathrm{~mm}$, reached for the displacements of the CoM in the frontal plane, followed by the transverse plane $(129.92 \mathrm{~mm})$ and the sagittal plane $(126.25 \mathrm{~mm})$. The analysis of mean values of angular ranges for this cluster demonstrated that the ankle joints in the sagittal plane reached the nearly symmetrical values i.e., $38.18^{\circ}$ (left) vs. $37.43^{\circ}$ (right). The ranges of motion in both hip joints in the frontal plane were $\approx 25^{\circ}$. The lowest values were found for the ankle joint in the frontal plane $\left(3^{\circ}\right)$.

The maximal mean for the cluster 2 (falling backwards) is $2506.41 \mathrm{~mm}$, observed for the CoM displacements in the sagittal plane. The analysis of mean values 
a)

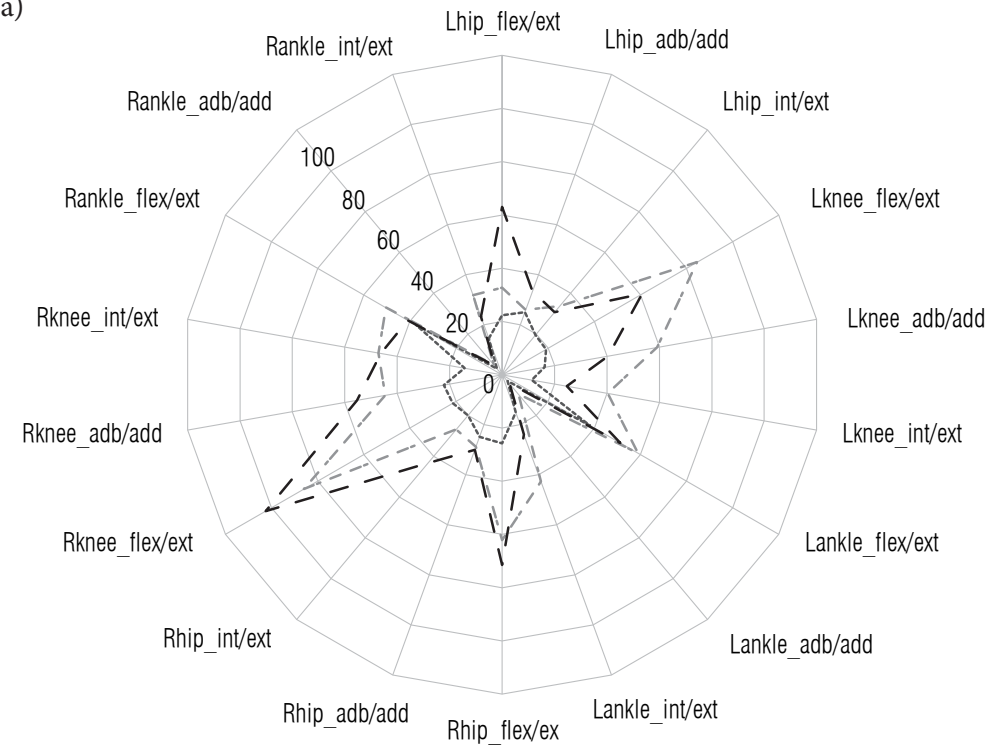

b)

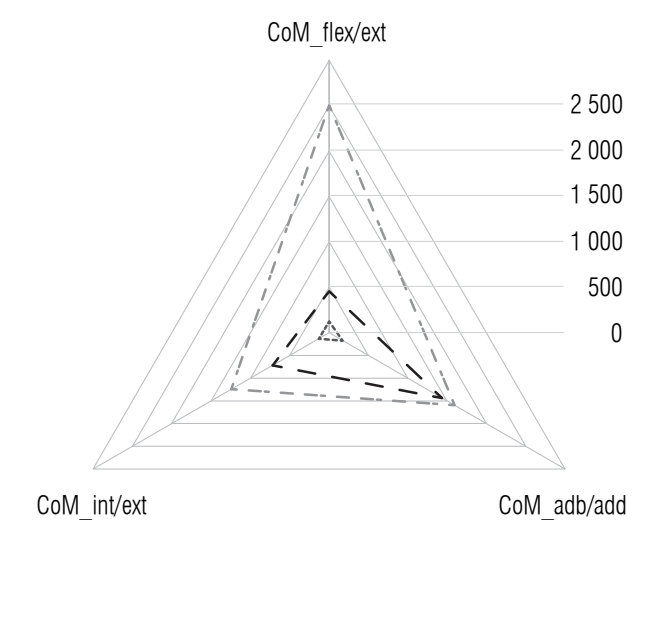

-.-..-... cluster 1

-..- - cluster 2

- - cluster 3

CoM - center of mass, $\mathrm{R}$ - right side of the body, L - left side of the body, flex/ext - flexion/extension, adb/add - abduction/adduction, int/ext - internal/external, $\mathrm{R}$ - Pearson's correlation coefficient.

Fig. 1. The 20-s test on the platform Biodex Balance System Static and Dynamic in the study group $(\mathrm{N}=20)$ : a) mean angular ranges of motion in joints of lower limbs, b) mean displacements of CoM

of angular ranges for this cluster reveals the biggest changes for the knee joints in the sagittal plane of $85^{\circ}$. The lowest values $\left(7^{\circ}\right)$ were recorded for the ankle joint in the frontal plane. Furthermore, the characterization for the cluster 3 (falling to the side) reveals that the highest means for the CoM displacements are obtained in the frontal plane $(1438.1 \mathrm{~mm})$, followed by those observed in the transverse plane $(716.52 \mathrm{~mm})$. The analysis of angular displacements demonstrated the great range of motion in the right knee joint in the sagittal plane $\left(102.49^{\circ}\right)$, which suggested a knee bend during a fall. Similar angle range $\left(67.27^{\circ}\right)$ was observed in the hip joints in the sagittal plane. Similar to the previous case, the smallest values $\left(4^{\circ}\right)$ were recorded for the ankle joint in the frontal plane.

The analysis above reveals that the differences in the CoM displacements in the sagittal plane and the angular values for the hip and knee joint in participants were the parameters which differentiated between the participants the most and determined the categorization of people into groups. Eleven participants who maintained stable position on the platform were assigned to the cluster 1 . Five people were assigned to the cluster 2 and only 4 persons formed the cluster 3 . Clusters 2 and 3 were characterized by substantial differences in $\mathrm{Ri}$ for the joints, leading to falls.

\section{Stability strategies}

The Shapiro-Wilk test used for the results obtained for the cluster 1 showed that stability indexes and all ranges of motion for kinematic parameters had normal distribution. The Table 1 presents the biggest values of Pearson's correlation coefficients between stability indexes and kinematic parameters.

The values contained in the Table 1 show that the highest correlation is observed between the displacements of the CoM in the sagittal plane (CoM flexion/ extension) and the stability index OSI. The high correlation coefficient was also found between APSI and motions in the left ankle joint in the sagittal plane.

The one-way ANOVA was performed to test differences between individual zones for each joint of lower limb. Significant differences were found for the variable hip (left and right) between means (I, II, III vs. IV) at $\mathrm{p}=0.0001$. For knee (I, II, III vs. IV), this value was $\mathrm{p}=0.0001$, for ankle - I vs. II: $\mathrm{p}=0.0317$, I vs. III: $\mathrm{p}=0.0002$ (Figure 2).

The results show that on an unstable surface, there are 3 strategies to maintain stability: ankle, knee and hip. The ankle strategy is adopted in the zone I, when maintaining balance does not show disturbances and the ankle joint movements are dominant (the highest frequency of access to the I zone). With greater postural 
Table 1. Correlations between the overall stability index (OSI), the anterior-posterior stability index (APSI), the medial-lateral stability index (MLSI) and range of motions for kinematics parameters within 20-s test on the platform Biodex Balance System Static in the study group $(\mathrm{N}=20)$

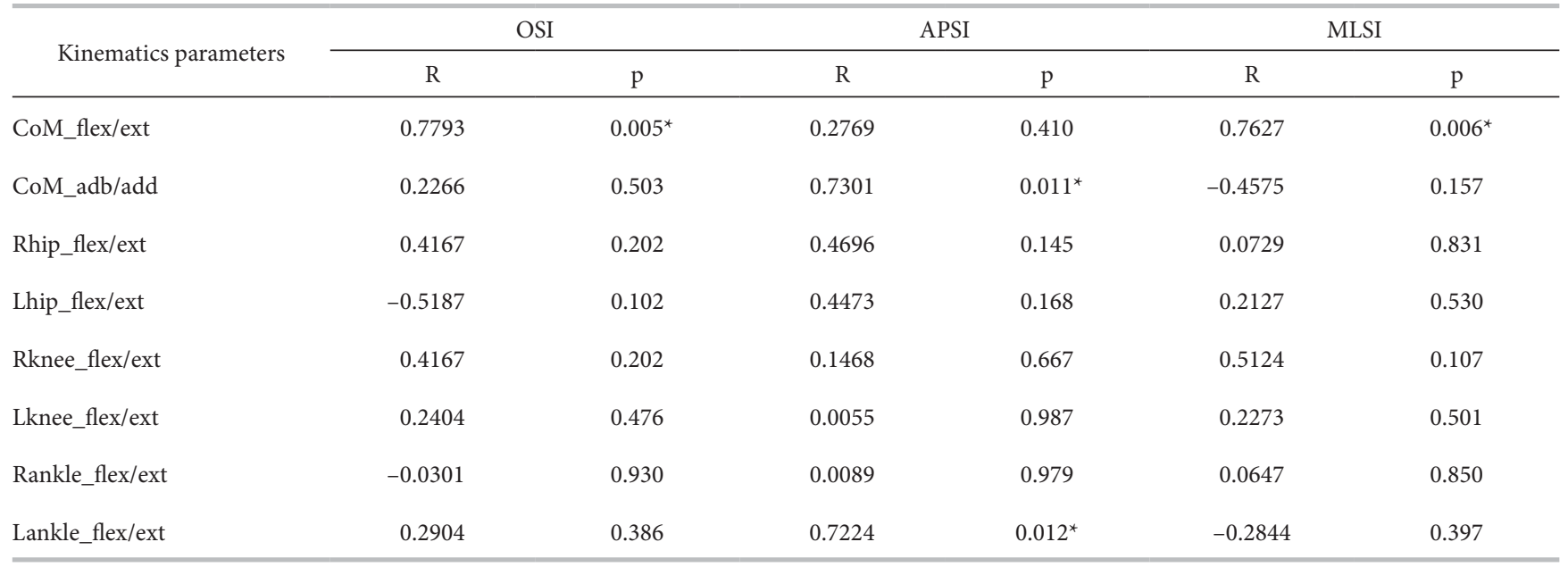

* Significant correlations at $\mathrm{p}<0.05$.

Abbreviations as in Figure 1.

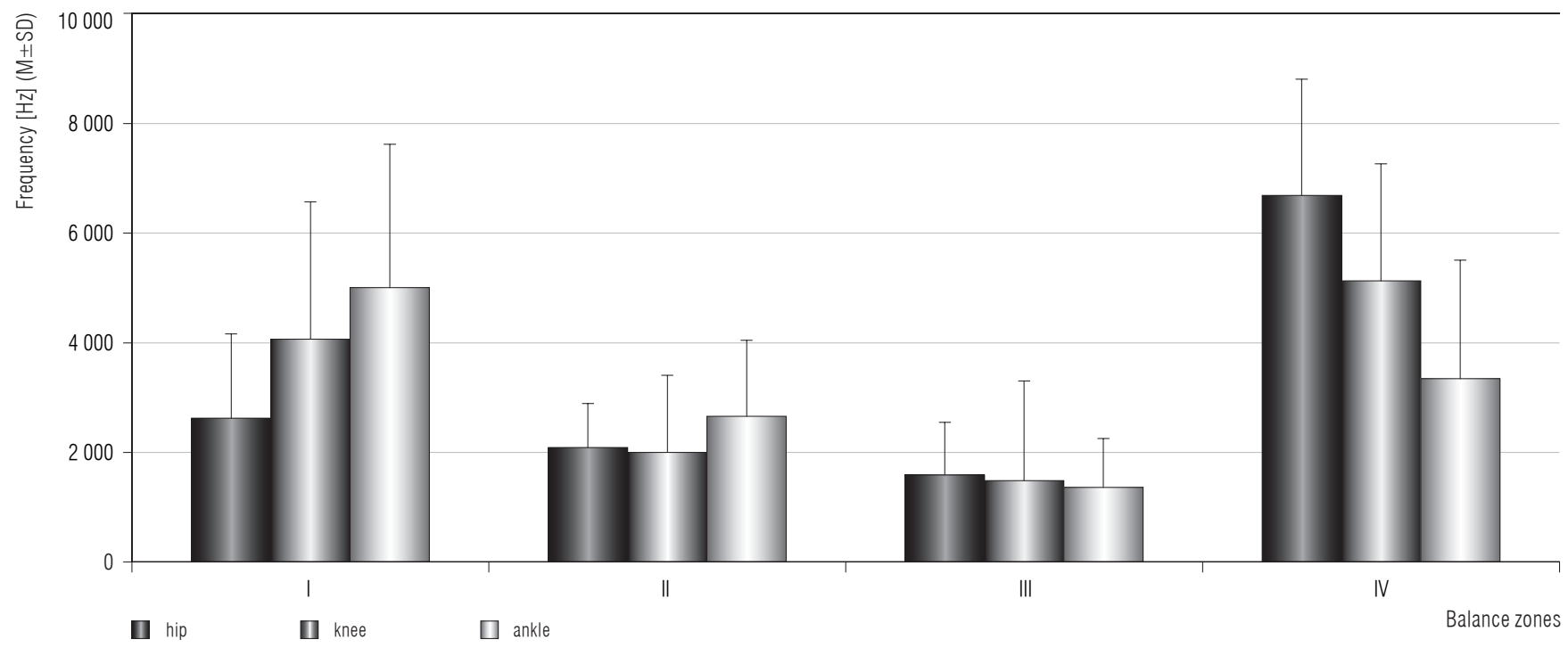

$\mathrm{M}$ - mean, SD - standard deviation.

Zone I: $(-5)-5^{\circ}$, zone II: $(-10)-(-5)^{\circ}$ and $5-10^{\circ}$, zone III: $(-15)-(-10)^{\circ}$ and $10-15^{\circ}$, zone IV: $(-\infty)-(-15)^{\circ}$ and $15^{\circ}-\infty$.

Fig. 2. Frequency of access to the balance zones by each of lower limb joint within 20-s test on the platform Biodex Balance System Static and Dynamic in the study group $(\mathrm{N}=20)$

sway, i.e., in the zones II and III, the similar ranges are observed for all joints. The biggest ranges are found for the zone IV, with the dominant joint being the hip, followed by the knee joint and then the ankle joint.

\section{DISCUSSION}

Falls from an unstable ground and from height represent the issue for forensic pathology and nowadays fo- rensic anthropology also recognizes the skeletal trauma evaluation in major blunt traumas as the key area. Therefore, this study has aimed to determine the parameters that describe human reactions at the instant of losing balance evoked by instability of the platform under the lower limbs. Furthermore, the attempts were made to determine the parameters that allowed for finding the strategies used during standing on an unstable ground. 
In the past, several systems [11-13] were used for assessing postural control. These devices typically used force plates to determine the displacements of the center of pressure (CoP). In contrast to force plate, the BBS, which was employed as a measurement tool in this study, measures the degree of tilt with respect to each axis during dynamic conditions rather than measuring the deviation of the CoP during static conditions, Thus, the BSS appears to provide more specific information on ankle joint movements. However, it is unclear how knee and hip motions affect BSS measures or how these measures relate to CoM fluctuations. The results presented in our study suggest that there are 2 types of strategies used by humans during standing on an unstable ground: falling or maintaining balance. The parameters which determine these behaviors are mainly displacements of the CoM in the frontal and sagittal planes. These displacements had the effect mainly on angular values for the hip and knee joints in the study participants.

Falls from height or unstable ground represent a specific type of blunt force trauma, which is produced by rapid vertical deceleration and impact forces $[2,8,14,15]$. Very few studies in the field of the occupational safety and health $[2,16]$ have been described in literature. Davis et al. [17] studied how standing at elevated surface heights and the associated changes in the visual field affected human balance control. When standing at the height of $3.2 \mathrm{~m}, 10$ out of 36 participants reported an increase in anxiety and a robust fear response while the remaining 26 participants only experienced an increase in anxiety and no fear response. The between-subject analysis of the effect of surface height on postural control revealed that fearful and non-fearful participants adopted different postural control strategies with increased heights. Human body behavior during a fall has been analyzed in just a few publications $[7,18,19]$.

Hsiao and Robinovitch [18] reported that any fall from height had the potential to cause hip fracture. But, only $1-2 \%$ of falls among the elderly result in hip fracture, and less than $10 \%$ cause serious injuries. This suggests that highly effective movement strategies exist for preventing injury. To determine the nature of these, the authors measured body segment movements as subjects stood upon a gymnasium mattress and attempted to prevent themselves from falling after the mattress was made to translate abruptly. Subjects were more than twice as likely to fall after anterior translations of the feet, when compared to posterior or lateral translations. In falls which resulted in the impact to the pelvis, a complex sequence of upper extremity movements allowed subjects to impact their wrists at nearly the same instant as the pelvis, suggesting shared contact energy between the 2 body parts. Finally, the marked trunk rotation was exhibited in falls due to lateral perturbations, resulting in the avoidance of impact to the lateral aspect of the hip.

In our study, out of 20 people standing on the most unstable BBS platform for $20 \mathrm{~s}, 9$ people fell, with 5 falling backwards and 4 to the side. As it could be expected, falling backward reaches the biggest values for the CoM displacements in the sagittal plane. Furthermore, the characterization for falling to the side revealed that the highest mean values for the CoM displacements were observed in the frontal plane $(1438.1 \mathrm{~mm})$, followed by those observed in the transverse plane $(716.52 \mathrm{~mm})$. This analysis shows that the differences in the values of the CoM displacements are the parameters that differentiate between the participants most in terms of the type and direction of the fall. Further involvement in terms of the range of motion during both types of falls was observed for the knee joints, followed by the hip joints and ankle joints.

These results suggest that body segment movements during falls, rather than being random and unpredictable, involve a repeatable series of responses which facilitate safe landing. Nevitt and Cummings [19] reported that those who suffered from hip fractures were more likely to have fallen sideways or straight down. Among persons who fell on the hip, those with hip fractures were taller, less likely to have landed on a hand or to break the fall by grabbing or hitting an object, had weaker triceps, and were more likely to land on a hard surface than those without fractures. Subjects with wrist fractures were more likely to have fallen backward and to have landed on a hand than those who fell without a fracture. Among people who fell on the hand, those with wrist fractures were taller and less likely to break the fall by grabbing or hitting an object. Therefore, the nature of the fall determines the type of fracture.

The areas of stability and falls are linked to specific strategies used by people in the process of postural control of the vertical body posture. In 1985, Nashner and McCollum hypothesized the existence of 2 discrete strategies that could either be used separately or be combined to produce adaptable control of the horizontal position of the CoM in the sagittal plane. The ankle strategy repositioned the CoM by moving the whole body as a single segment inverted pendulum 
by production of torque at the ankle [20]. The ankle joint strategy is a distal-proximal sequence, performed at insignificant disturbance of the vertical body position. It is observed during an insignificant disturbance of balance for people standing on the stable ground. The hip strategy is a proximal-distal sequence and, in contrast, body moves as a double segment inverted pendulum with counter phase motion at the ankle and hip.

Moreover, this strategy should be observed in situations that limit the effectiveness of ankle torque at producing whole-body motion. Horak et al. [21] examined the strategies used for the stable ground, while our study verified these observations under unstable conditions. In order to determine the strategy applied to maintain stability on the tilting platform, the angular motion was divided into 4 zones: I, II, III and IV [9]. With $20 \mathrm{~s}$ of the test performed for each joint, the number of times the joint remained within the zones I, II, III or IV was counted. Our data suggests that ankle strategy dominates in the zone I $(-5)-5^{\circ}$ whereas body sway is insignificant.

Similar findings were documented by Boyas et al. [22], with participants standing on the stable ground after fatigue. Participants increased the flexion of the ankle and/or the hip joints to compensate for the effects of fatigue, but conserved the ankle strategy as the dominant postural strategy in both planes. With regard to time spent in IV zone, our findings indicate hip strategy. The CoM displacements were high, with very high risk of falling. With ground instability, the person has to avoid a fall over the whole duration of the test, which also leads to fatigue. The studies by Amori et al. [23] have demonstrated that the pelvis is used as a reference frame for the body balance in postural control of healthy adults exposed to continuous multi-axial support surface perturbation. It seems that the above study is well confirmed by Wang et al. [12]. Therefore, fall prevention should focus on stabilization of pelvis and hip joints as these body parts seem to be the most critical to prevention of falls in the workplace. Therefore, balance training is recommended for all workers who work on the unstable ground to prevent falls.

\section{CONCLUSIONS}

1. Fall prevention continues to be a considerable challenge across the care continuum.

2. Knowledge of human reactions during falls is essential from the standpoint of both prevention and limitation of the consequences of falls.
3. The findings obtained in the study help anticipate the reaction of a worker in dangerous situations.

4. The obtained results may help develop and implement technological and organizational solutions that are likely to substantially improve workers' safety.

\section{REFERENCES}

1. Hung YH, Winchester WW, 3rd, Smith-Jackson TL, Kleiner BM, Babski-Reeves KL, Mills TH, 3rd. Identifying fallprotection training needs for residential roofing subcontractors. Appl Ergon. 2013;44:372-80, https://doi.org/10. 1016/j.apergo.2012.09.007.

2. Casali MB, Battistini A, Blandino A, Cattaneo C. The injury pattern in fatal suicidal falls from a height: An examination of 307 cases. Forensic Sci Int. 2014;244:57-62, https://doi.org/10.1016/j.forsciint.2014.08.004.

3. Talbot LA, Musiol RJ, Witham EK, Metter EJ. Falls in young, middle-aged and older community dwelling adults: Perceived cause, environmental factors and injury. BMC Public Health. 2005;5:86, https://doi.org/10.1186/14712458-5-86.

4. Milanowicz M, Budziszewski P, Kędzior K. Experimental study of the loss of balance process before falling from a height. Acta Bioeng Biomech. 2016;18:55-64.

5. Fass S, Yousef R, Liginlal D, Vyas P. Understanding causes of fall and struck-by incidents: What differentiates construction safety in the Arabian Gulf region? Appl Ergon. 2017;58:515-26, https://doi.org/10.1016/j.apergo.2016. 05.002 .

6. Hyde AS. Accidental falls: Their causes and their injuries: Fundamentals of slipping, tripping, stumbling, tumbling, and crumpling. Key Biscayne: HAI; 1996.

7. Maki B, McIlroy W, Perry S. Influence of lateral destabilization on compensatory stepping responses. J Biomech. 1996;29(3):343-53, https://doi.org/10.1016/0021-9290(95) 00053-4.

8. Lapostolle F, Gere C, Borron SW, Petrovic T, Dallemagne F, Beruben A, et al. Prognostic factors in victims of falls from height. Crit Care Med. 2005;33:1239-42, https://doi. org/10.1097/01.CCM.0000164564.11989.C3.

9. Arnold BL, Schmitz RJ. Examination of balance measures produced by the biodex stability system. J Athl Train. 1998;33:323-7.

10. Glave AP, Didier JJ, Weatherwax J, Browning SJ, Fiaud V. Testing postural stability: Are the Star Excursion Balance Test and Biodex Balance System Limits of Stability Tests consistent? Gait Posture. 2016;43:225-7, https://doi.org/ 10.1016/j.gaitpost.2015.09.028. 
11. Runge CF, Shupert CL, Horak FB, Zajac FE. Ankle and hip postural strategies defined by joint torques. Gait Posture. 1999;10:161-70, https://doi.org/10.1016/S0966-6362 (99)00032-6.

12. Wang LY, Liaw MY, Huang YC, Lau YC, Leong CP, Pong YP, et al. Static and dynamic balance performance in patients with osteoporotic vertebral compression fracture. J Back Musculoskelet Rehabil. 2013;26:199-205, https:// doi.org/10.3233/BMR-130369.

13. Wiszomirska I, Kaczmarczyk K, Blażkiewicz M, Wit A. The impact of a vestibular-stimulating exercise regime on postural stability in people with visual impairment. Biomed Res Int. 2015;2015:136969, https://doi.org/10.1155/2015/ 136969.

14. Bush J. Viability of virtual reality exposure therapy as a treatment alternative. Comput Hum Behav. 2008;24: 1032-40, https://doi.org/10.1016/j.chb.2007.03.006.

15. Robinovitch SN, Hayes WC, McMahon TA. Prediction of femoral impact forces in falls on the hip. J Biomech Eng. 1991;113:366-74, https://doi.org/10.1115/1.2895414.

16. Isbister ES, Roberts JA. Autokabalesis: A study of intentional vertical deceleration injuries. Injury. 1992;23:119-22, https://doi.org/10.1016/0020-1383(92)90046-U.

17. Davis JR, Campbell AD, Adkin AL, Carpenter MG. The relationship between fear of falling and human postural control. Gait Posture. 2009;29:275-9, https://doi.org/10.1016/ j.gaitpost.2008.09.006.
18. Hsiao ET, Robinovitch SN. Common protective movements govern unexpected falls from standing height. J Biomech. 1998;31:1-9, https://doi.org/10.1016/S0021-92 90(97)00114-0.

19. Nevitt MC, Cummings SR. Type of fall and risk of hip and wrist fractures: The study of osteoporotic fractures. J Am Geriatr Soc. 1993;41:1226-34, https://doi.org/10.11 11/j.1532-5415.1993.tb07307.x.

20. Nashner LM, McCollum G. The organization of human postural movements: A formal basis and experimental synthesis. Behav Brain Sci. 1985;8:135-50, https://doi.org/10. 1017/S0140525X00020008.

21. Horak FB, Nashner LM, Diener HC. Postural strategies associated with somatosensory and vestibular loss. Exp Brain Res. 1990;82:167-77, https://doi.org/10.1007/BF00230848.

22. Boyas S, Hajj M, Bilodeau M. Influence of ankle plantarflexor fatigue on postural sway, lower limb articular angles, and postural strategies during unipedal quiet standing. Gait Posture. 2013;37:547-51, https://doi.org/10.1016/j.gait post.2012.09.014.

23. Amori V, Petrarca M, Patane F, Castelli E, Cappa P. Upper body balance control strategy during continuous 3D postural perturbation in young adults. Gait Posture. 2015;41: 19-25, https://doi.org/10.1016/j.gaitpost.2014.08.003.

This work is available in Open Access model and licensed under a Creative Commons Attribution-NonCommercial 3.0 Poland License - http://creativecommons.org/licenses/by-nc/3.0/pl/deed.en. 